\title{
Capecitabine combined with (-)-epigallocatechin-3-gallate inhibits angiogenesis and tumor growth in nude mice with gastric cancer xenografts
}

\author{
HONGJU WU ${ }^{1}$, YAN XIN ${ }^{2}$, CHONGAN XU $^{1}$ and YUPING XIAO ${ }^{2}$ \\ ${ }^{1}$ Department of Oncology Medicine, The Fourth Affiliated Hospital of China Medical University, \\ Shenyang, Liaoning 110032; ${ }^{2}$ Fourth Laboratory of the Cancer Institute, \\ The First Affiliated Hospital of China Medical University, Shenyang, Liaoning 110001, P.R. China
}

Received November 14, 2011; Accepted January 3, 2012

DOI: $10.3892 /$ etm.2012.448

\begin{abstract}
Low-dose metronomic chemotherapy represents a new strategy to treat solid tumors by exhibiting stronger anti-angiogenic activity and less side effects, especially in combination with other anti-angiogenic agents. Capecitabine is a novel fluoropyrimidine carbamate, which has a broader spectrum of antitumor activity than other fluoropyrimidines, such as 5-FU, DFUR or UFT; it has proved effective over a wide dose range. The aim of this study was to investigate the anti-angiogenic effect of capecitabine alone and combined with the angiogenic inhibitor (-)-epigallocatechin-3-gallate (EGCG) on gastric cancer. A BGC-823 human gastric cancer xenograft model was used, and tumor growth, side effects and the number of days of survival of mice were closely monitored and recorded. Quantitative real-time PCR was used to determine vascular endothelial growth factor (VEGF) mRNA levels. The expression of VEGF and CD31 was determined by immunohistochemistry. Our results indicated that metronomic capecitabine inhibited angiogenesis, growth of gastric cancer and improved survival with less toxicity, and the effects were further enhanced by the concurrent administration of EGCG. Clinical trials and further pre-clinical studies, will hopefully provide answers to the use of continuous low-dose anti-angiogenic therapies for the treatment of human gastric cancer.
\end{abstract}

\section{Introduction}

Gastric cancer is a highly prevalent disease with an impact on morbidity and mortality. Despite the progress in cancer therapeutics and chemotherapy development with the introduction

Correspondence to: Dr Hongju Wu, Department of Oncology Medicine, The Fourth Affiliated Hospital of China Medical University, Shenyang, Liaoning 110032, P.R. China

E-mail: zgydwhj@163.com

Key words: capecitabine,(-)-epigallocatechin-3-gallate, angiogenesis, gastric cancer, metronomic chemotherapy of new drugs, advanced gastric cancer continues to have an extremely poor prognosis and limited treatment options (1). Thus, new therapeutic strategies are urgently required. Tumor endothelium is a new target for treatments. A number of recent studies have sparked interest in the notion of exploiting chemotherapeutic drugs as angiogenesis inhibitors. Maximum tolerated dose (MTD) chemotherapy, targeting the tumor cells directly, sometimes causes undesirable side effects normally associated with traditional cytotoxic chemotherapy regimens. Continuous, low-dose metronomic (LDM) chemotherapy, targeting the endothelial cells directly, is thought to have fewer side effects as well as a lack of drug resistance (2). Also, metronomic chemotherapy is changing the paradigm that more is better (3).

Capecitabine, an oral fluoropyrimidine designed to selectively deliver 5-fluorouracil (5-FU) to tumor cells, is not inferior to 5-FU and it has a more convenient and comfortable administration method. Capecitabine has been widely used in advanced gastric cancer, either in monotherapy or in combination (4). Even though capecitabine shows higher specificity to tumor cells, many late-phase gastric cancer patients cannot tolerate its side effects of diarrhea, nausea, lymphopenia and hand-foot syndrome; furthermore, drug resistance often occurs in the final stages. We postulated that low-dose capecitabine may have anti-angiogenic effects on gastric cancer. In this study, we evaluated its efficacy in impeding tumor growth and microvasculature in gastric cancer.

The effects of LDM chemotherapy can be improved by concurrent administration of a drug targeting angiogenesis $(5,6)$. The rationale for this combination is that these targeted drugs, capable of specific blockade of activated endothelial cell survival mechanisms, selectively enhance the damaging or cytotoxic effects of LDM chemotherapy on newly formed blood vessels (7). Green tea extract and its major component (-)-epigallocatechin-3-gallate (EGCG) possess obvious antiproliferative, pro-apoptotic and anti-angiogenic effects (8). EGCG is an agent which exhibits anti-angiogenic activities through a variety of mechanisms $(9,10)$. Several studies have suggested that EGCG may reduce the toxicity of certain anticancer drugs and can be used in adjuvant settings for cancer treatment (8). 
Therefore, we designed this study to investigate the efficacy of LDM chemotherapy with capecitabine, alone or in combination with EGCG, in a nude mouse model of BGC-823 human gastric carcinoma tumors.

\section{Materials and methods}

Materials. The BGC-823 human gastric cancer cell line was obtained from the Cancer Research Institution of China Medical University (China). A group of female BALB/c nude mice (6-8 weeks of age) weighing 18-20 g were purchased from the Animal Experiment Center of Beijing, China. Mice were housed under pathogen-free conditions and fed with animal chow and water ad libitum. Capecitabine (N-pentyloxycarbonyl-5'-deoxy-5-fluorocytidine) was obtained from Roche Pharmaceutical Company (Nutley, NJ, USA). EGCG was obtained from Shanghai Winherb Medical Company, China. SYBR Green reagents were purchased from Takara Bio (Japan). Human monoclonal antibody for vascular endothelial growth factor (VEGF) was purchased from Fuzhou Maixin Company (China). Mouse monoclonal antibody for CD31 was purchased from Dako (Japan).

Cell culture. Human BGC-823 gastric cancer cells were cultured in Roswell Park Memorial Institute medium (RPMI-1640) supplemented with $10 \%$ fetal bovine serum plus ampicillin and streptomycin routinely, and incubated in $5 \% \mathrm{CO}_{2}$ at $37^{\circ} \mathrm{C}$.

Design of animal experiments. All of the animal experiments were approved by the Institutional Animal Care and Use Committee of China Medical University. BGC-823 cells $\left(2 \times 10^{6}\right)$ were suspended in $0.2 \mathrm{ml}$ of phosphate-buffered saline (PBS) and injected into the right flank of each BALB/c nude mice. Approximately 2 weeks later (average tumor size $\left.100 \mathrm{~mm}^{3}\right)$, the mice were randomized into five groups $(\mathrm{n}=10)$ as follows: control [intraperitoneal (i.p.) injection of $0.2 \mathrm{ml}$ physiological saline daily]; MTD $(500 \mathrm{mg} / \mathrm{kg}$ capecitabine daily by gavage for 2 weeks, followed by a 1-week rest period); LDM (received $200 \mathrm{mg} / \mathrm{kg}$ capecitabine daily by gavage); EGCG (i.p. injection of $1.5 \mathrm{mg}$ EGCG daily) and the combined therapy with LDM capecitabine and EGCG at doses identical to the single agent.

Tumor growth and side effects. The mice were closely monitored and body weight and tumor size were recorded twice a week. Tumor volume (TV) was estimated using the formula: $\mathrm{TV}\left(\mathrm{mm}^{3}\right)=\left(\right.$ width $^{2} \mathrm{x}$ length $) / 2$. Blood samples were collected through orbital sinus once a week to count white blood cells (WBC). During the experiment period, side effects, such as weight loss, change in behavior and feeding, reaction to stimulation and ruffling of fur, were observed. When a mouse died, the number of days of survival was recorded.

Real-time polymerase chain reaction (PCR) analysis. The frozen BGC-823 xenografts were collected and total RNA was extracted from each xenograft by TRIzol method (Invitrogen, USA). RNA was reverse-transcribed with oligo (dT) primers and mRNA expression was analyzed by quantitative realtime reverse transcription-PCR using the following primers: VEGF sense 5'-GAGCCTTGCCTTGCTGCTCTAC and antisense 5'-CACCAGGGTCTCGATTGGATG. The relative expression of each gene was assessed in comparison to the housekeeping gene $\beta$-actin amplified with the following primers: sense 5'-TGGCACCCAGCACAATGAA and antisense 5'-CTAAGTCATAGTCCGCCTAGAAGCA. cDNAs were amplified for 45 cycles with a PCR Thermal Cycler (Takara Bio) containing the intercalating agent SYBR Green in a two-step amplification scheme $\left(95^{\circ} \mathrm{C}, 5 \mathrm{sec}\right.$ and $\left.60^{\circ} \mathrm{C}, 30 \mathrm{sec}\right)$. Amplifications were normalized to $\beta$-actin and the quantitation of gene expression was performed using the ${ }^{\Delta \Delta} \mathrm{Ct}$ calculation, where $\mathrm{Ct}$ is the threshold cycle; the amount of target, normalized to the endogenous control and relative to the calibrator (vehicle-treated control tumors), is given as $2^{-\Delta \Delta C t}$.

Immunohistochemical detection of VEGF and CD31. Tumor tissues were fixed immediately in $10 \%$ buffered formalin phosphate and embedded in paraffin. Sections $(5 \mu \mathrm{m})$ were cut for immunohistochemistry using the PV-9000 kit (Beijing Zhongshan Goldenbridge Biotechnology Company, Beijing, China). Rabbit anti-human monoclonal antibody for VEGF (dilution 1:50) was purchased from Fuzhou Maixin Company (China). Rat anti-mouse monoclonal antibody for CD31 (dilution 1:100) was purchased from Dako (Japan). Antigens were retrieved after they were placed in a pressure cooker at a full pressure for $160 \mathrm{sec}$ in citrate buffer ( $\mathrm{pH}$ 6.0). All procedures were implemented according to the manufacturer's instructions, respectively. For the negative controls, sections were processed as above, but treated with $0.01 \mathrm{~mol} / 1 \mathrm{PBS}$ instead of the primary antibodies.

Cytoplasmic staining was scored positive for VEGF. The degree of positivity was evaluated by calculating the percentage of immunoreactive cells on a minimum of 500 cells (11).

Microvessel density (MVD) was assessed by immunohistochemical analysis with antibodies to the endothelial marker CD31 and determined according to the method of Liu et al (12). Briefly, the immunostained sections were initially screened at low magnifications (x40 and x100) to identify hot spots, which are the areas of highest neovascularization. Any yellow brown-stained endothelial cell or endothelial cell cluster that was clearly separate from adjacent microvessels, tumor cells and other connective tissue elements was considered a single, countable microvessel. Within the hot spot area, the stained microvessels were counted in a single high-power (x200) field, and the average vessel count in three hot spots was considered the value of MVD. All counts were performed by three investigators in a blinded manner. Microvessel counts were compared between the observers, and the discrepant results were reassessed. The consensus was used as the final score for analysis.

Statistical analysis. Survival data were evaluated by KaplanMeier survival analysis and other data were analyzed by ANOVA, followed by the Student-Newman-Keuls test. All statistical analyses were performed using SPSS 17.0 software package. Data are expressed as the means \pm standard error. P-values $<0.05$ were considered significant.

\section{Results}

Tumor-growth assessment. As shown in Fig. 1, in mice treated with sterile saline (control group), tumors grew promptly - all 


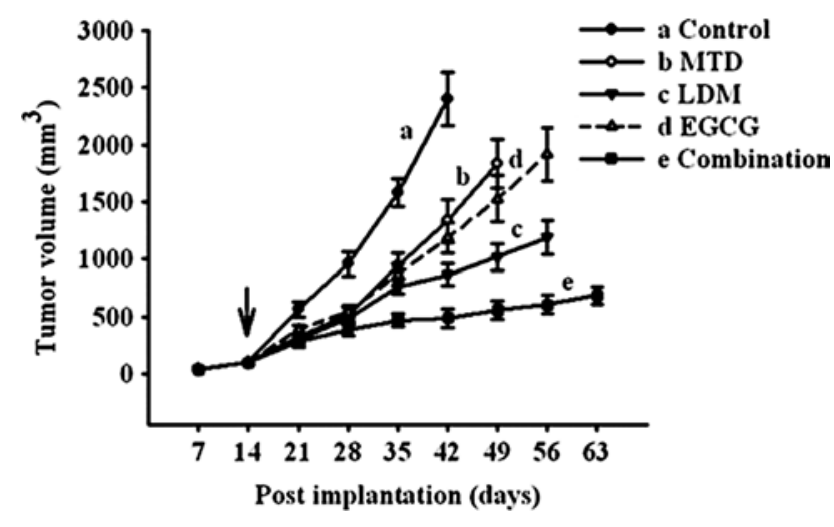

Figure 1. Tumor-growth curve of each group. BGC-823 gastric tumors were established in BALB/c nude mice. Two weeks later, when the size of the tumors reached $\sim 100 \mathrm{~mm}^{3}$ (indicated by a vertical arrow), mice were assigned into five groups: Control, MTD, LDM, EGCG and Combination (LDM + EGCG). Tumor growth was inhibited in mice receiving conventional MTD capecitabine at the prophase, after which tumors grew fast while on therapy. For mice receiving continuous LDM capecitabine, tumor growth was delayed for a long period of time. When LDM capecitabine was combined with EGCG, tumor growth delays were more remarkable and enduring.

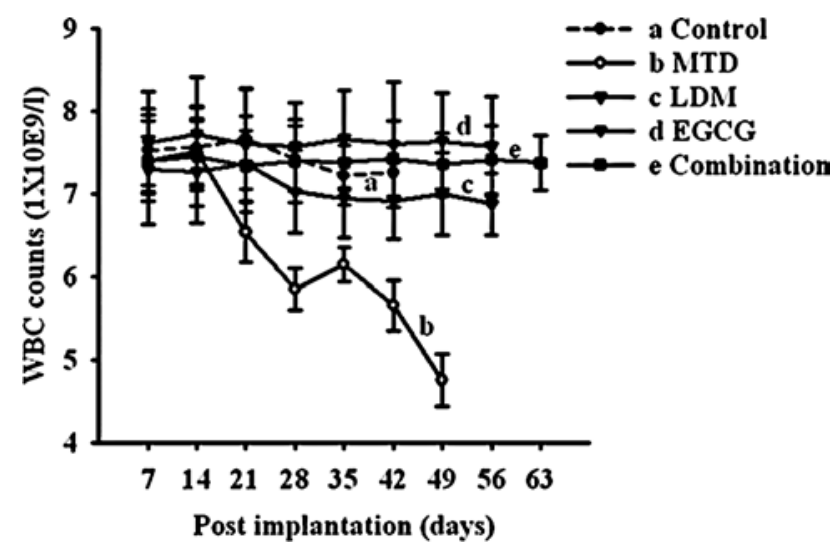

Figure 2. White blood cell (WBC) counts of BALB/c nude mice in each group. The five groups of mice had similar WBC counts in the beginning, but 2 weeks after initiation of antitumor therapy, conventional MTD chemotherapy produced significant leucopenia compared to the other four groups $(\mathrm{P}<0.05)$. The continuous LDM capecitabine and EGCG regimens were not associated with leucopenia, and the two-drug combination was also tolerable. $\mathrm{P}<0.05$ vs. the other groups.

the animals had large tumors after 3 weeks. Compared to the control, tumor growth in mice treated with MTD capecitabine was remarkably delayed for the first 2 weeks, after which tumors began to grow progressively while on therapy. Notably, when LDM capecitabine was administered continuously, tumor growth was delayed compared to the MTD group when assessed after 3 weeks of treatment $(\mathrm{P}<0.05$; Fig. 1$)$. EGCG retarded tumor growth to much the same extent as MTD capecitabine ( $P>0.05$; Fig. 1). Combined therapy with metronomic capecitabine and EGCG resulted in tumorgrowth delays that were more marked and enduring than those resulting from either of them when used alone $(\mathrm{P}<0.05$; Fig. 1).

Evaluation of toxicity. Mice treated with the conventional MTD regimen produced severe side effects, consisting of weight loss, leukopenia and skin discoloration. As shown in Fig. 2, the five groups of mice had similar WBC counts in the

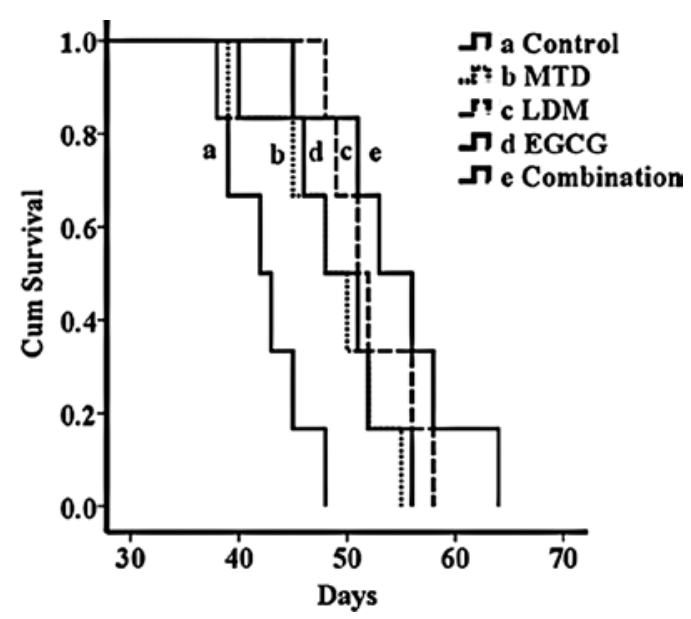

Figure 3. Kaplan-Meier survival analyses of mice bearing gastric tumors. MTD capecitabine, LDM capecitabine, EGCG and combined therapy all prolonged the survival time of mice bearing gastric tumors $(\mathrm{P}<0.05)$, but they showed no significant difference with each other $(\mathrm{P}>0.05)$.

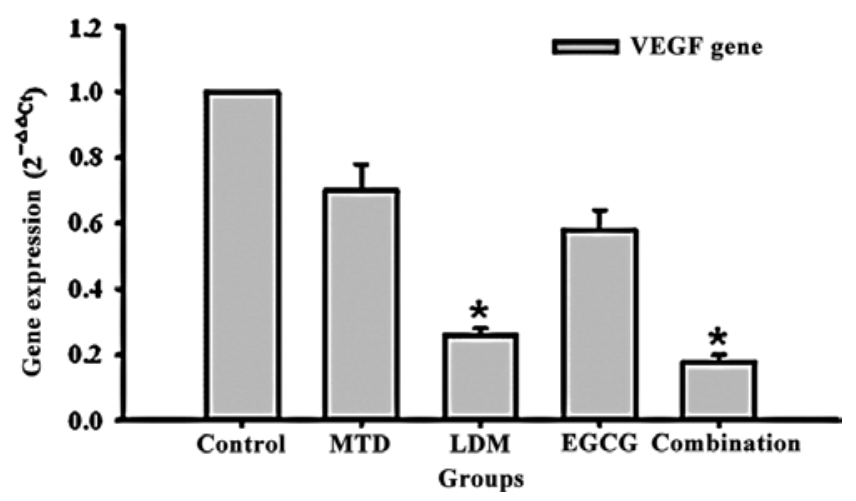

Figure 4. Expression levels of VEGF mRNA in untreated or drugadministered xenograft tumor tissues. Each real-time PCR was carried out in duplicate. The gene values were normalized to the level of $\beta$-actin in the respective tissues. ${ }^{*} \mathrm{P}<0.05$ vs. the vehicle-treated controls.

beginning, but 2 weeks after initiation of antitumor therapy, a significant decrease in WBC counts was observed in the MTD group ( $\mathrm{P}<0.05$; Fig. 2). The continuous LDM capecitabine regimen, EGCG regimen and the combined therapy with LDM capecitabine and EGCG were not associated with weight loss, leucopenia or other signs of toxicity.

Survival analysis. Median survival time for mice in the control group was 42 days. Treatment with MTD was able to improve median overall survival to 48 days $(\mathrm{P}<0.05$; Fig. 3). Mice receiving continuous LDM capecitabine presented a median overall survival of 51 days, at no significant difference, when compared to the conventional MTD regimen ( $\mathrm{P}>0.05$; Fig. 3). Mice treated with continuous LDM capecitabine combined with EGCG showed almost the same survival time as those treated with either of them when used alone ( $>0.05$; Fig. 3).

Real-time PCR analysis. Fig. 4 shows the modulation of human VEGF gene expression in the treated tumors compared to the vehicle-treated control tumors. The VEGF expression in the LDM capecitabine-treated group and combination group was lower than that in the control group (both $\mathrm{P}<0.05$; Fig. 4). 
Table I. Results of MVD and VEGF in immunohistochemical staining.

\begin{tabular}{lcc}
\hline Group & $\begin{array}{c}\text { MVD } \\
\overline{\mathrm{x}} \pm \mathrm{s}(\mathrm{n} / \mathrm{x} 200)\end{array}$ & $\begin{array}{c}\text { VEGF } \\
\overline{\mathrm{x}} \pm \mathrm{s}(\%)\end{array}$ \\
\hline Control & $30.48 \pm 2.87$ & $78.43 \pm 6.52$ \\
MTD capecitabine & $20.13 \pm 1.76^{\mathrm{a}}$ & $54.38 \pm 3.16^{\mathrm{a}}$ \\
LDM capecitabine & $10.57 \pm 1.08^{\mathrm{b}}$ & $41.79 \pm 2.93^{\mathrm{b}}$ \\
EGCG & $10.64 \pm 1.31^{\mathrm{b}}$ & $50.02 \pm 4.75^{\mathrm{a}}$ \\
Combination & $4.01 \pm 1.49^{\mathrm{c}}$ & $16.41 \pm 2.52^{\mathrm{c}}$ \\
\hline
\end{tabular}

${ }^{\mathrm{a}} \mathrm{P}<0.05$ vs. the control group; ${ }^{\mathrm{b}} \mathrm{P}<0.05$ vs. the MTD group; ${ }^{\mathrm{C}}<0.05$ vs. the LDM and EGCG groups.

The VEGF expression in the MTD and EGCG groups also was lower than that in the control group, however, without significant difference ( $\mathrm{P}>0.05$; Fig. 4).

Histopathologic analysis. A comparison of angiogenic indices revealed some variance among different treatment groups (Table I). Tumors derived from mice in the control group showed the highest microvessel counts, whereas those from mice subjected to the MTD group had comparatively lower microvessel counts $(\mathrm{P}<0.05$; Table I). The LDM or EGCG group had lower microvessel counts than the MTD group $(\mathrm{P}<0.05$; Table I). However, the lowest counts in the combination group were measured $(\mathrm{P}<0.05$; Table I). There was positive expression of VEGF in the cytoplasm of certain tumor cells. Compared to the MTD group, VEGF expression was lower in the continuous LDM capecitabine group $(\mathrm{P}<0.05$; Table I). When EGCG was added to continuous LDM capecitabine treatment, the indicators were much lower $(\mathrm{P}<0.05$; Table I). The results indicated that metronomic capecitabine chemotherapy inhibited tumor angiogenesis and its anti-angiogenic effect was further improved when combined with EGCG.

\section{Discussion}

Our data, for the first time, showed that LDM capecitabine, used alone or combined with EGCG, is effective in pre-clinical settings of gastric cancer, as an anti-angiogenic and antitumor schedule, modulating VEGF gene expression.

LDM chemotherapy may offer several advantages over the MTD approach: i) it may significantly delay the onset of acquired drug resistance; ii) it may facilitate long-term coadministration of different chemotherapeutic drugs lowering toxicity and adverse side effects; iii) more importantly, it seems that despite the lower cumulative doses administered, it is superior to MTD-based regimens for inhibiting tumor growth in pre-clinical models and clinical trials (13-15). Until recently, little was known about the anti-angiogenic effect of capecitabine in gastric cancer. Our findings show that metronomic capecitabine had significant effects on therapeutic response and survival. LDM chemotherapy is less likely to acquire resistance compared to conventional chemotherapy, since the target of the therapy is presumed to be genetically stable and activated endothelial cells rather than genetically unstable cancer cells (16). In our study, tumor growth was delayed at the prophase in mice receiving MTD capecitabine, after which the tumors began to grow progressively, which could be explained by drug resistance. However, when LDM capecitabine was administered, the regimen produced a long period of growth delay in tumor mass.

An attractive application of metronomic chemotherapy is the ability to combine these regimens with biological agents, in particular anti-angiogenic drugs, and the effects of these schedules may be further enhanced $(17,18)$. Such combinations are particularly appealing as high local concentrations of VEGF in the tumor environment promote multidrug resistance in tumor endothelium $(14,19,20)$. In our study, metronomic capecitabine plus EGCG led to a substantial tumor reduction over the respective monotherapies. Moreover, we did not observe toxic effects at the doses administered in the LDM and combinational groups, which remained similar to those of control mice during the treatment period.

LDM was more effective at inhibiting tumor growth, which may be attributed to its anti-angiogenic effects. Angiogenesis is tightly regulated by pro-angiogenic and anti-endothelial growth factors. Recent advances in the knowledge of tumor angiogenesis have shed light on the pivotal role of VEGF (21), and VEGF is an important mediator of angiogenesis; it is a potent endothelial cell mitogen, morphogen and vascular permeability-inducing agent (22). MVD is accepted as a standard indicator of angiogenesis (23), and VEGF expression is strictly correlated with MVD (24). In the present study, MTD capecitabine and EGCG did not significantly change the expression levels of VEGF mRNA; instead, the VEGF mRNA expression in the LDM and combination groups decreased significantly. Indeed, MVD in tumor xenografts was markedly decreased in metronomic schedules when compared to the control group, and also to the MTD regimen, which were in agreement with the findings of Bocci et al (11) and Ji et al (25). In addition, MVD in the combination group decreased significantly compared to the LDM group. VEGF protein expression in tumors was similar to that of MVD. Our data indicated that LDM chemotherapy exhibited the inhibitory effects of angiogenesis by decreasing MVD value and VEGF expression. The effects were improved by concurrent administration of EGCG.

Both LDM and MTD capecitabine increased the life span of mice implanted with BGC-823 gastric cancer cells, with no significant difference, when compared to each other. EGCG, given alone or combined with LDM capecitabine, was also well tolerated and prolonged the survival time of mice.

Our data indicate that LDM capecitabine and EGCG may potentiate each other's antitumor and anti-angiogenesis activities. The mechanism responsible for the interaction between LDM capecitabine and EGCG remains unclear. On one hand, EGCG modulates the expression of multidrug resistance proteins (26). Moreover, studies have shown the inhibitory effect of EGCG on angiogenic growth and transcription factors, VEGF and hypoxia-inducible factor-1 (HIF-1) (27). On the other hand, EGCG and green tea are potent inhibitors of neutrophil-mediated angiogenesis in vitro and in vivo (28). EGCG also inhibited VEGF-induced endothelial cell proliferation, migration and tube formation (29). In addition, EGCG has been shown to modulate growth-factor signaling pathways and, additionally, has effects on cell-cycle progression and 
tumor cell invasion (30). The above effects may contribute to the synergistic inhibition of tumor angiogenesis and tumor growth. However, additional effort is required to fully understand the exact mechanisms.

Taken together, LDM capecitabine chemotherapy alone or its combination with EGCG inhibited angiogenesis, growth of gastric cancer and improved survival with less toxicity in a nude mouse model of BGC-823 gastric cancer. Clinical trials and further pre-clinical studies will hopefully provide answers regarding the use of continuous low-dose anti-angiogenic therapies for the treatment of malignancies.

\section{Acknowledgements}

The study was supported by the National Natural Science Foundation of China (nos. 30973503,81071650 and 81050007), and the Research Fund for the Doctoral Program of Higher Education (20092104110008).

\section{References}

1. Albert A: New drugs in the treatment of gastric tumors. Clin Transl Oncol 10: 256-261, 2008.

2. Orlando L, Cardillo A, Rocca A, et al: Prolonged clinical benefit with metronomic chemotherapy in patients with metastatic breast cancer. Anticancer Drugs 17: 961-967, 2006.

3. Scharovsky OG, Mainetti LE and Rozados VR: Metronomic chemotherapy is changing the paradigm that more is better. Curr Oncol 16: 7-15, 2009.

4. Tham CK, Choo SP and Poon DY: Capecitabine with radiation is an effective adjuvant therapy in gastric cancers. World J Gastroenterol 16: 3709-3715, 2010.

5. Bergers $G$ and Hanahan D: Combining antiangiogenic agents with metronomic chemotherapy enhances efficacy against latestage pancreatic islet carcinomas in mice. Cold Spring Harb Symp Quant Biol 67: 293-300, 2002.

6. Garcia AA, Hirte H, Fleming G, et al: Phase II clinical trial of bevacizumab and low-dose metronomic oral cyclophosphamide in recurrent ovarian cancer: a trial of the California, Chicago, and Princess Margaret Hospital phase II consortia. J Clin Oncol 26 76-82, 2008

7. Klement G, Baruchel S, Rak J, et al: Continuous low-dose therapy with vinblastine and VEGF receptor-2 antibody induces sustained tumor regression without overt toxicity. J Clin Invest 105: R15-R24, 2000

8. Khan N, Afaq F, Saleem M, Ahmad N and Mukhtar H: Targeting multiple signaling pathways by green tea polyphenol (-)-epigallocatechin-3-gallate. Cancer Res 66: 2500-2505, 2006.

9. Neuhaus T, Pabst S, Stier S, et al: Inhibition of the vascularendothelial growth factor-induced intracellular signaling and mitogenesis of human endothelial cells by epigallocatechin-3 gallate. Eur J Pharmacol 483: 223-227, 2004.

10. Fassina G, Vene R, Morini M, et al: Mechanisms of inhibition of tumor angiogenesis and vascular tumor growth by epigallocatechin-3-gallate. Clin Cancer Res 10: 4865-4873, 2004.

11. Bocci G, Falcone A, Fioravanti A, et al: Antiangiogenic and anticolorectal cancer effects of metronomic irinotecan chemotherapy alone and in combination with semaxinib. Br J Cancer 98 : $1619-1629,2008$

12. Liu TG, Huang Y, Cui D, et al: Inhibitory effect of ginsenoside $\mathrm{Rg} 3$ combined with gemcitabine on angiogenesis and growth of lung cancer in mice statistical analysis. BMC Cancer 9: 250, 2009 .
13. Kamat AA, Kim TJ, Landen CN, et al: Metronomic chemotherapy enhances the efficacy of antivascular therapy in ovarian cancer. Cancer Res 67: 281-288, 2007.

14. Kerbel RS and Kamen BA: The anti-angiogenic basis of metronomic chemotherapy. Nat Rev Cancer 4: 423-436, 2004.

15. Benelli R, Monteghirfo S, Balbi C, Barboro P and Ferrari N: Novel antivascular efficacy of metronomic docetaxel therapy in prostate cancer: hnRNP $\mathrm{K}$ as a player. Int J Cancer 124: 2989-2996, 2009.

16. Bocci G, Nicolaou KC and Kerbel RS: Protracted low-dose effects on human endothelial cell proliferation and survival in vitro reveal a selective antiangiogenic window for various chemotherapeutic drugs. Cancer Res 62: 6938-6943, 2002.

17. Zhang M, Tao W, Pan S, Sun X and Jiang H: Low-dose metronomic chemotherapy of paclitaxel synergizes with cetuximab to suppress human colon cancer xenografts. Anticancer Drugs 20: 355-363, 2009.

18. Zhang Q, Kang X and Zhao W: Antiangiogenic effect of low-dose cyclophosphamide combined with ginsenoside $\mathrm{Rg} 3$ on Lewis lung carcinoma. Biochem Biophys Res Commun 342: 824-828, 2006.

19. Kerbel RS: Inhibition of tumor angiogenesis as a strategy to circumvent acquired resistance to anticancer therapeutic agents. Bioessays 13: 31-36, 1991

20. Castilla MA, Caramelo C, Gazapo RM, et al: Role of vascular endothelial growth factor (VEGF) in endothelial cell protection against cytotoxic agents. Life Sci 67: 1003-1013, 2000.

21. Ma YP, Yang Y,Zhang S, et al: Efficient inhibition of lung cancer in murine model by plasmid-encoding VEGF short hairpin RNA in combination with low-dose DDP. J Exp Clin Cancer Res 29: $56,2010$.

22. Machado DE, Berardo PT, Palmero CY, et al: Higher expression of vascular endothelial growth factor (VEGF) and its receptor VEGFR-2 (Flk-1) and metalloproteinase-9 (MMP-9) in a rat model of peritoneal endometriosis is similar to cancer diseases. J Exp Clin Cancer Res 29: 4, 2010.

23. Magnon C, Galaup A, Rouffiac V, et al: Dynamic assessment of antiangiogenic therapy by monitoring both tumoral vascularization and tissue degeneration. Gene Ther 14: 108-117, 2007.

24. Raspollini MR, Castiglione F, Garbini F, et al: Correlation of epidermal growth factor receptor expression with tumor microdensity vessels and with vascular endothelial growth factor expression in ovarian carcinoma. Int J Surg Pathol 13: 135-142, 2005.

25. Ji Y, Hayashi K, Amoh Y, et al: The camptothecin derivative CPT-11 inhibits angiogenesis in a dual-color imageable orthotopic metastatic nude mouse model of human colon cancer. Anticancer Res 27: 713-718, 2007.

26. Mei Y, Qian F, Wei D and Liu J: Reversal of cancer multidrug resistance by green tea polyphenols. J Pharm Pharmacol 56: 1307-1314, 2004.

27. Domingo DS, Camouse MM, Hsia AH, et al: Anti-angiogenic effects of epigallocatechin-3-gallate in human skin. Int J Clin Exp Pathol 3: 705-709, 2010.

28. Donà M, Dell'Aica I, Calabrese F, et al: Neutrophil restraint by green tea: inhibition of inflammation, associated angiogenesis, and pulmonary fibrosis. J Immunol 15: 4335-4341, 2003.

29. Zhu BH, Zhan WH, Li ZR, et al: (-)-Epigallocatechin-3-gallate inhibits growth of gastric cancer by reducing VEGF production and angiogenesis. World J Gastroenterol 13: 1162-1169, 2007.

30. Lang M, Henson R, Braconi C and Patel T: Epigallocatechingallate modulates chemotherapy-induced apoptosis in human cholangiocarcinoma cells. Liver Int 29: 670-677, 2009. 